\title{
Erratum to: An integer linear programming formulation and heuristics for the minmax relative regret robust shortest path problem
}

\author{
Amadeu Almeida Coco ${ }^{1}$ • João Carlos Abreu Júnior ${ }^{1}$ • Thiago F. Noronha ${ }^{1}$. \\ Andréa Cynthia Santos ${ }^{2}$
}

Published online: 18 March 2017

(C) Springer Science+Business Media New York 2017

\section{Erratum to: J Glob Optim (2014) 60:265-287 DOI 10.1007/s10898-014-0187-x}

Let the critical scenario $r$ of a path $P \subseteq A$ from $s \in V$ to $t \in V$ be the one, among all the possible scenarios, in which arc costs are set to $c_{i j}^{r}=u_{i j}$ for all $(i, j) \in P$, and $c_{i j}^{r}=l_{i j}$ for all $(i, j) \in A \backslash P$. The authors in [3] proved that the maximum regret of $P$ over all scenarios occurs in its critical scenario. Therefore, the maximum regret of $P$ is given by $R_{P}=C_{P}^{r}-C_{S}^{r}$, where $S$ is the shortest path between $s$ and $t$ in the scenario $r$, and $C_{P}^{r}=\sum_{(i, j) \in P} c_{i j}^{r}$ and $C_{S}^{r}=\sum_{(i, j) \in S} c_{i j}^{r}$ are the costs of $P$ and $S$ in the scenario $r$.

This work focuses on finding the minmax relative regret of $P$ in critical scenarios, as it is an intuitive bad situation [2]. The problem addressed aims at finding the path $P \subseteq A$ from $s$ to $t$ with the smallest value of $\widehat{R}_{P}=\frac{C_{P}^{r}-C_{S}^{r}}{C_{S}^{r}}=\frac{C_{P}^{r}}{C_{S}^{r}}-1$. This robust optimization criterion has been studied by other authors in the scientific literature $[1,4,5]$. The motivation of using the minmax relative regret over the minmax regret criterion can be found in Sect. 1. The study considers that the maximum relative regret of $P$ occurs in its critical scenario, as it

The online version of the original article can be found under doi:10.1007/s10898-014-0187-x.

Thiago F. Noronha

tfn@dcc.ufmg.br

Amadeu Almeida Coco

amadeuac@dcc.ufmg.br

João Carlos Abreu Júnior

joao.junior@dcc.ufmg.br

Andréa Cynthia Santos

andrea.duhamel@utt.fr

1 Departamento de Ciência da Computação, Universidade Federal de Minas Gerais, UFMG, Avenida Antônio Carlos 6627, Belo Horizonte, MG CEP 31270-901, Brazil

2 ICD-LOSI, Université de Technologie de Troyes, 12, rue Marie Curie, CS 42060, 10004 Troyes Cedex, France 
is the case for other robust optimization criteria. However, we show below that this is not necessarily true. Nevertheless, we prove that the version of the robust shortest path problem studied is NP-Hard and point out that this version of the problem is suitable to applications sensitive to critical scenarios.

Proposition 1 the maximum relative regret of $P$ does not necessarily occur in its critical scenario.

Proof The proof is given by a counterexample. Figure 1 shows an example of a Karasan instance [3] with six vertices and a path $P=[s, 1,3, t]$ depicted with a dotted line. Figure 2 depicts the critical scenario $r$ induced by $P$. The relative regret of $P$ in $r$ is $\frac{(40+30+10)}{(2+3+10)}-1$ $=4.33$. However, if the cost of arc $(3, t) \in P$ is set to 1 , as shown in Fig. 3, one can obtain a scenario where the relative regret of $P$ increases to $\frac{(40+30+1)}{(2+3+1)}-1=10.83$, despite the fact that the cost of $P$ is smaller in this scenario. Therefore, the maximum relative regret of $P$ does not necessarily occur in its critical scenario.

In order to prove that the minmax relative regret in critical scenarios is NP-Hard, we first prove Lemma 1. This result is then used in the proof of Proposition 2 below. Let $P_{1}$ and $P_{2}$ be any two paths from $s$ to $t$, and $r_{1}$ and $r_{2}$ be the critical scenarios of $P_{1}$ and $P_{2}$, respectively. Besides, let also $S_{1}$ and $S_{2}$ be the shortest path from $s$ to $t$ in the scenarios $r_{1}$ and $r_{2}$, respectively.

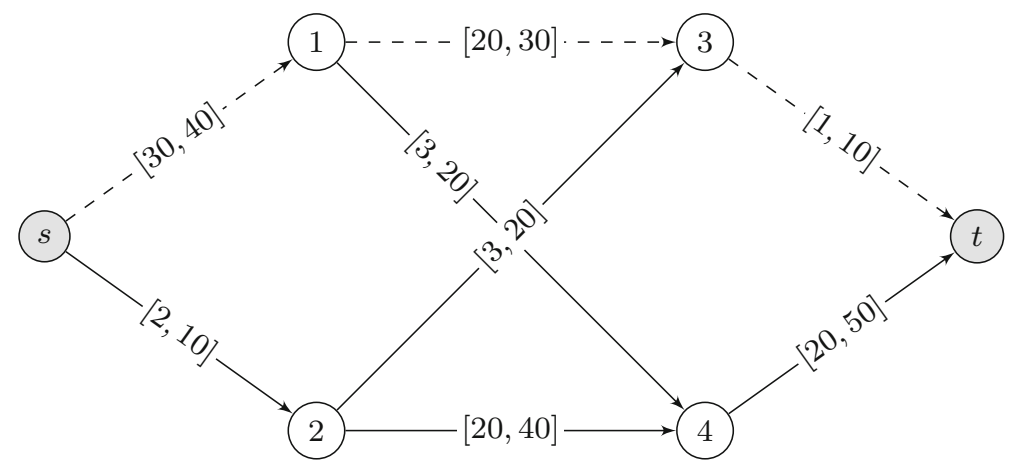

Fig. 1 Karasan instance with a dashed path $P$

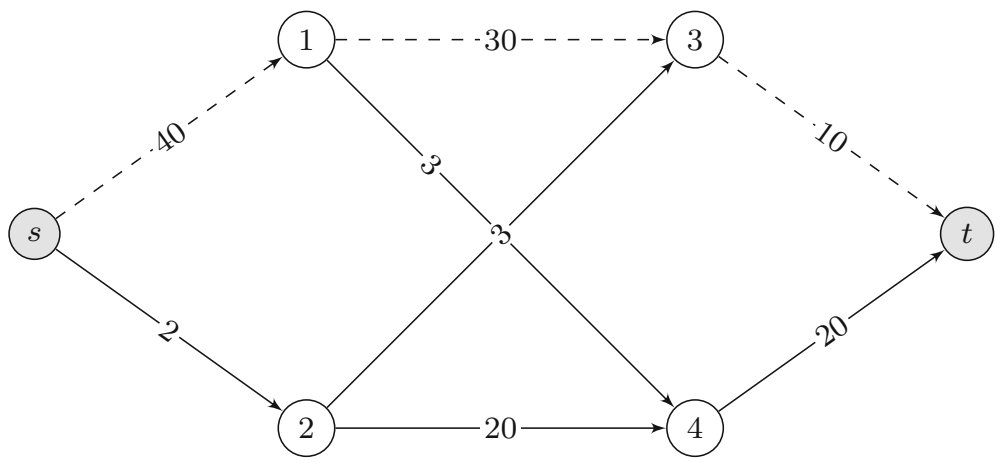

Fig. 2 Critical scenario induced by $P$ 


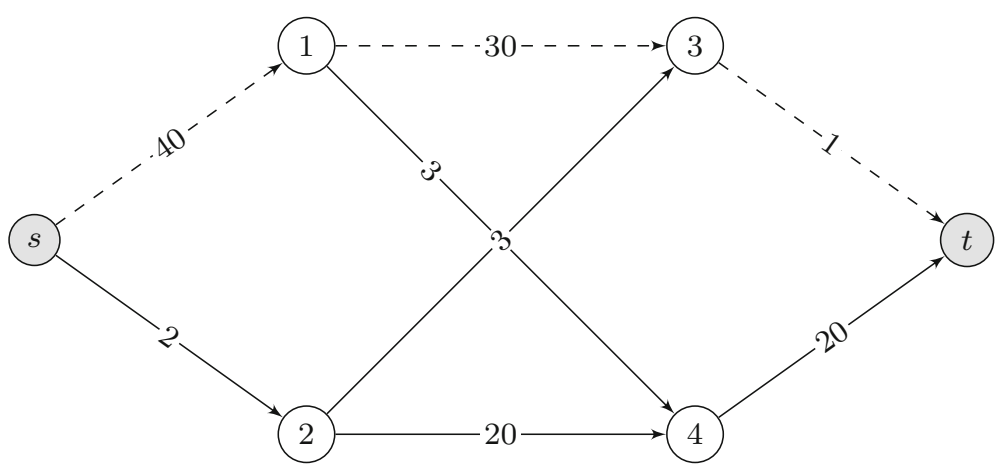

Fig. 3 An arbitrary scenario where the relative regret of $P$ is larger than the one in its critical scenario

Lemma 1 Given two paths $P_{1}$ and $P_{2}$, Eq. (1) holds for $M \geq(|V| \cdot U)^{2}$, where $U=\max _{(i, j) \in A} u_{i j}$.

$$
C_{P_{2}}^{r_{2}}-C_{S_{2}}^{r_{2}}>C_{P_{1}}^{r_{1}}-C_{S_{1}}^{r_{1}} \Longrightarrow \frac{C_{P_{2}}^{r_{2}}+M}{C_{S_{2}}^{r_{2}}+M}>\frac{C_{P_{1}}^{r_{1}}+M}{C_{S_{1}}^{r_{1}}+M},
$$

Proof We have that

$$
\frac{C_{P_{2}}^{r_{2}}+M}{C_{S_{2}}^{r_{2}}+M}-\frac{C_{P_{1}}^{r_{1}}+M}{C_{S_{1}}^{r_{1}}+M}=\frac{\left(C_{S_{1}}^{r_{1}}+M\right) \cdot\left(C_{P_{2}}^{r_{2}}+M\right)-\left(C_{S_{2}}^{r_{2}}+M\right) \cdot\left(C_{P_{1}}^{r_{1}}+M\right)}{\left(C_{S_{2}}^{r_{2}}+M\right) \cdot\left(C_{S_{1}}^{r_{1}}+M\right)},
$$

which can be rewritten as (2).

$$
\frac{M \cdot\left(C_{P_{2}}^{r_{2}}-C_{S_{2}}^{r_{2}}-C_{P_{1}}^{r_{1}}+C_{S_{1}}^{r_{1}}\right)+C_{P_{2}}^{r_{2}} \cdot C_{S_{1}}^{r_{1}}-C_{P_{1}}^{r_{1}} \cdot C_{S_{2}}^{r_{2}}}{\left(C_{S_{2}}^{r_{2}}+M\right) \cdot\left(C_{S_{1}}^{r_{1}}+M\right)}
$$

From the left-hand side of (1), we have that $C_{P_{2}}^{r_{2}}-C_{S_{2}}^{r_{2}}-C_{P_{1}}^{r_{1}}+C_{S_{1}}^{r_{1}}>0$. Besides, we have by definition that $l_{i j} \in \mathbb{Z}_{+}^{*}$ and $u_{i j} \in \mathbb{Z}_{+}^{*}$ for all $(i, j) \in A$. Therefore, Eq. (3) holds.

$$
C_{P_{2}}^{r_{2}}-C_{S_{2}}^{r_{2}}-C_{P_{1}}^{r_{1}}+C_{S_{1}}^{r_{1}} \geq 1 .
$$

Equation (3) together with $M \geq(|V| \cdot U)^{2}>C_{P_{1}}^{r_{1}} \cdot C_{S_{2}}^{r_{2}}$ implies that (2) is greater than zero, which completes the proof.

Proposition 2 Solving the minmax relative regret $R S P$ in the critical scenarios is NP-hard.

Let $f:\{\langle G, l, u, s, t\rangle\} \mapsto\left\{\left\langle G^{\prime}, l, u, s^{\prime}, t\right\rangle\right\}$ be a function that transforms an instance $I$ of minmax regret RSP in an instance $\widehat{I}$ of minmax relative regret RSP in critical scenarios, as defined by (4)-(7), where $M=(|V| \cdot U)^{2}$.

$$
\begin{aligned}
& V^{\prime}=V \cup\left\{s^{\prime}\right\} \\
& A^{\prime}=A \cup\left\{\left(s^{\prime}, s\right)\right\} \\
& G^{\prime}=\left(V^{\prime}, A^{\prime}\right) \\
& l_{\left(s^{\prime}, s\right)}=u_{\left(s^{\prime}, s\right)}=M
\end{aligned}
$$

The worst case complexity of the transformation $I \rightarrow \hat{I}$ is clearly polynomial. Given an optimal solution $\widehat{P}_{2} \subseteq A$ to $\widehat{I}$, the path $P_{2}=\widehat{P}_{2} \backslash\left(s^{\prime}, s\right)$ is also optimal for $I$. Otherwise, 
there would exist a solution $P_{1}$ to $I$ with lower robust cost than the one of solution $P_{2}$. However, from Lemma $1, \widehat{P}_{1}=P_{1} \cup\left(s^{\prime}, s\right)$ would have a robust relative cost smaller than $\widehat{P}_{2}$, which is a contradiction. Therefore, $P_{2}$ is indeed optimal for $I$.

\section{References}

1. Averbakh, I.: Computing and minimizing the relative regret in combinatorial optimization with interval data. Discrete Optim. 2, 273-287 (2005)

2. Coco, A.A., Júnior, J.C.A., Noronha, T.F., Santos, A.C.: An integer linear programming formulation and heuristics for the minmax relative regret robust shortest path problem. J. Glob. Optim. 60(2), 265-287 (2014)

3. Karasan, O.E., Yaman, H., Ç. Pinar, M.: The robust shortest path problem with interval data. Tech. rep., Bilkent University, Department of Industrial Engineering (2001)

4. Kouvelis, P., Yu, G.: Robust Discrete Optimization and Its Applications. Kluwer Academic Publishers, Dordrecht (1997)

5. Mausser, H.E., Laguna, M.: Minimising the maximum relative regret for linear programming with interval objective function coefficients. J. Oper. Res. Soc. 50, 1063-1070 (1999) 